\title{
LA INNOVACIÓN: UN PROCESO SOCIAL E INTERACTIVO BASADO EN EL CONOCIMIENTO
}

\author{
Ferneliy Rutz Gómez ${ }^{1}$
}

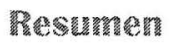

El presente artículo es un análisis crítico que trata de mostrar la necesidad de concebir la innovación como un proceso social e interactivo basado en el conocimiento, que impacta la sociedad a nivel cognitivo, organizacional y económico. Esta concepción es necesaria si un determinado gobierno pretende una mejor gestión de la innovación, potenciando el desarrollo social y económico por medio de políticas públicas en la materia. El escrito es un avance del trabajo investigativo adelantado en el programa de Doctorado en Estudios sobre ciencia y tecnología y, Gestión de la innovación tecnológica, servido por la Universidad del País Vasco.

Gestionar mejor la innovación implica enfrentarse a la creciente especialización en la producción de conocimiento, el aumento de la complejidad en los artefactos físicos, así como a las fuertes dificultades para hacer corresponder las oportunidades tecnológicas con las necesidades del mercado y las prácticas organizacionales. Este complejo mosaico hace necesario analizar la existencia e interacción de los agentes desde el concepto de Sistema de Innovación, considerando que son sistemas abiertos, dinámicos y sociales, como consecuencia de las interacciones que se producen entre los agentes socioeconómicos que lo constituyen y con el entorno.

Además, la introducción de nuevos agentes y la generación de nuevos tipos de interacciones son características relevantes en la dinámica de los sistemas, asunto que ha llevado a la necesidad de hablar de Innovation Networks o Redes de Innovación.

1 Director de la Escuela de Egresados del ITM. Ingeniero Electricista, Especialista en Telemática y estudiante de Doctorado en Gestión de la Innovación Tecnológica con la Universidad del País Vasco. E-mail: fernellyruiz@itm.edu.co 


\section{Palaluras cllave}

Innovación, Sistemas de innovación, Redes de innovación.

\section{Ahsirac}

This is a critical analysis in order to show the necessity of conceiving the innovation like a social and interactive process based on the knowledge, which impacts the society cognitively, organizationally and economically. This conception is necessary to be adopted if a certain government wants to achieve a better innovation management, by increasing the social and economic development by means of political public in the matter. The article origin is part of an investigative work done in advanced in the PHD program on Technological Innovation Management offered by the Universidad del País Vasco -UPV.

A better management innovation implies to face the growing specialization of knowledge production, the increasing of in the physical devices complexity, as well as to the strong difficulties to make technological opportunities coincide with the market necessities and the organizational practices. This complex mosaic creates the necessity to analyze the agents' interaction under the Innovation System concept, considering that they are open, dynamic and social systems, as a consequence of the interactions produced between socioeconomic agents and their surroundings.

Moreover, the introduction of new agents and the generation of new types of interactions are one of the main characteristics of dynamic systems; this topic has created the necessity to talk about Innovation Networks.

\section{Key worts}

Innovation, Innovation Systems, Innovation Networks. 


\section{WTraveruccion}

Concebir la innovación como mejoras en productos o procesos y considerarla básicamente como un resultado de la actividad empresarial, es una reducción que lesiona gravemente el diseño y el desarrollo de políticas públicas en esta materia. Este error limita la visión de un Estado que pretenda fortalecer el tejido productivo y social bajo su responsabilidad.

En este sentido, el presente artículo es el análisis crítico sobre la necesidad de concebir la innovación en un sentido más amplio y complejo. Argumenta que asumirla como un proceso social e interactivo basado en el conocimiento permite identificar elementos de juicio necesarios para abrir nuevos caminos de construcción de conocimiento y potenciarlos por medio del diseño y el desarrollo de políticas públicas.

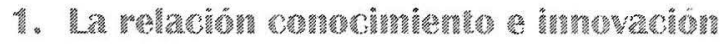

Es paradójico, no obstante, que ante los avances científicos y la proliferación de teorías con respecto a la innovación, ésta permanece en medida indescriptible y difícil de gestionar, variando de una gran sociedad a otra, de una región a otra, dependiendo además del sector y del tamaño de las empresas involucradas.

Este fenómeno llama la atención en el mundo y motiva a los investigadores en el tema a tratar de identificar elementos para su mejor gestión. Por ejemplo, Keith Pavitt (2003) del SPRU ${ }^{2}$ argumenta que del proceso innovativo se desprenden al menos tres subprocesos que se sobreponen entre sí: Producción de conocimiento; transformación de conocimiento en productos, servicios, procesos y sistemas; y la adaptación frente a las necesidades y demandas futuras del mercado. Asegura además este autor que el desarrollo de estos subprocesos y su superposición impactan a la sociedad en tres niveles: cognitivo, organizacional y económico.

2 Science and Technology Policy Research, England: Susexx University. 
Ante este mosaico potencialmente confuso, Pavitt rescata dos características generales sobre el proceso:

- Éste envuelve la exploración y explotación de oportunidades para obtener productos, servicios, procesos y/o sistemas nuevos o mejorados, basándose también en una ventaja técnica ("Know how"), un cambio en la demanda del mercado o una combinación de ambas.

- La incertidumbre es inherente a la innovación, lo que imposibilita predecir exactamente el costo y la construcción de un artefacto novedoso, así como las reacciones de los usuarios ante la novedad. Esta incertidumbre involucra inevitablemente procesos de aprendizaje cruzados por experimentación (ensayo y error) y de mejor entendimiento (teoría).

Más aún, dado que una parte (no toda) de dicho aprendizaje es específica a cada empresa, la competencia en el mercado capitalista puede ser considerada como un proceso de experimentación intencionada, compitiendo por la aceptación de los usuarios entre diferentes alternativas de productos, procesos y servicios, además de unos procesos técnicos y organizativos deliberadamente adoptados para tal fin. Así las cosas, la relación entre conocimiento e innovación se traduce en una interacción entre empresas y tecnología con implicaciones como:

- Una especialización y profesionalización con base en la naturaleza del conocimiento, razón por la cual el futuro de las empresas es condicionado por su aprendizaje en el pasado.

- Las empresas especializadas en diferentes campos del conocimiento tienden a destacar diferentes rasgos del proceso de innovación, dependiendo del campo donde se desempeña.

- El proceso de innovación difiere significativamente entre grandes y pequeñas empresas, a pesar del incremento paulatino de la relación entre las grandes corporaciones y las Pymes, mediante una desintegración vertical de la producción. 
Ahora, la innovación se desarrolla siguiendo diferentes trayectorias históricas y tecnológicas, razones para analizar la dinámica y las interacciones de los agentes socioeconómicos a través del tiempo, comprender y definir con mayor precisión la forma como evoluciona un tejido productivo, así como para detectar los cambios producidos por el diseño e implementación de las Políticas de Innovación. A esta característica evolutiva de un sistema productivo se le conoce como dependencia de la trayectoria o Path Dependent (Carlsson y Jacobson, 1997; Edquist ed., 1997).

Esta dependencia contribuye a la caracterización de la innovación en las empresas por el entorno productivo y social en donde se desarrollan y, por lo tanto, constituye un asunto no dependiente exclusivamente de ellas.

Pero a pesar de la singularidad de cada evolución histórica y tecnológica, se han identificado ciertas tendencias claves en el mundo, que acentúan el carácter social e interactivo de la innovación; éstas son: a) una creciente especialización en la producción de conocimiento; b) un aumento de la complejidad en los artefactos físicos y en las áreas de conocimiento que intervienen en su producción y, consecuentemente; c) fuertes dificultades en las empresas para corresponder las oportunidades tecnológicas con las necesidades del mercado y las prácticas organizacionales (Pavitt, 2003). Analicemos cada una de ellas.

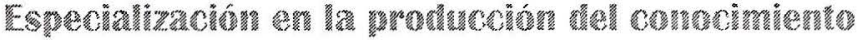

En relación con la especialización del conocimiento empresarial, se ha generado una tendencia al desarrollo de laboratorios de I+D en las grandes empresas, dedicados fundamentalmente a la producción de conocimiento para la explotación comercial; además, el surgimiento de una cantidad de pequeñas empresas que proveen mejoramientos continuos en la producción de bienes especializados.

A este intrincado panorama se suma una combinación de conocimiento de origen privado aplicado en los negocios de las 
empresas y conocimiento desarrollado en el sector público, generalmente diseminado por universidades e instituciones similares. Estas formas de especialización del conocimiento se presentan simultáneamente en un patrón de cambio técnico heterogéneo, dependiente de la trayectoria histórica, científica y tecnológica que resalta el matiz interactivo de la innovación y que, por supuesto, es difícil de gestionar.

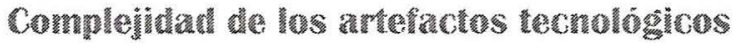

El amplio rango de campos del conocimiento que contribuyen al diseño y construcción de cada producto incrementa la complejidad de los artefactos, generándose otra tendencia, la modularización e integración en los sistemas productivos. De ahí, la importancia de las arquitecturas modulares en los productos, donde los componentes de interfaz son estandarizados e interdependientes, obligando a los nodos productivos de un sistema a transferirse información codificada trabajando juntos (interactuado) en relación con el mercado.

Cabe aclarar que la complejidad de los artefactos no se supera solamente codificando información, porque los campos del conocimiento que intervienen avanzan a diferentes velocidades. Esto significa que la división del trabajo entre empresas no debe ser vista como equivalente a una división de la labor en conocimiento; es preciso una superposición entre las empresas y sus correspondientes competencias para facilitar la transferencia de conocimiento tácito, con las consecuencias y los desbalances a que haya lugar, incrementándose así las dificultades en el diseño e integración entre sistemas productivos.

Además, los avances científicos incrementan la complejidad de los artefactos, situación generadora de acciones en doble dirección, mejoras en la calidad de la experimentación y reducción de los costes involucrados en la innovación. Esta generación se ha sumado entonces al aumento en la complejidad de los componentes, partes o subsistemas en un producto o servicio. Surge en consecuencia, otro asunto paradójico, en contraposición a los espectaculares. 
incrementos del conocimiento científico, la teoría es insuficiente para guiar la práctica tecnológica debido al incremento en la complejidad de los artefactos y de los conocimientos fundamentales en su construcción.

\section{Correspondencia con las demandas y oportunthades del mercalo}

Con respecto a esta tercera tendencia, uno de los mayores retos de la innovación en las empresas exitosas es la correspondencia de productos, procesos, sistemas, servicios y prácticas organizacionales, con las demandas del mercado y la demanda potencial. Esto es, los más importantes procesos por gestionar son aquellos que responden a las necesidades y demandas del mercado y, de este modo, corresponder las prácticas organizacionales con las oportunidades tecnológicas; gestión que implica enfrentarse con las consecuencias negativas de la especialización del conocimiento, y a cambios que implican rupturas con los paradigmas prevalecientes en las empresas.

Es la razón por la que uno de los más importantes factores de éxito empresarial es el grado de colaboración y de retroalimentación entre el diseño de producto y otras funciones corporativas, especialmente la manufactura y el mercadeo. Aunque se conocen buenas prácticas para lograr esta coordinación, el contacto personal permite la transferencia de elementos tácitos, los procesos informales permiten la retroalimentación. En definitiva, no se dispone de una descripción del proceso que asegure una efectiva correspondencia entre las oportunidades tecnológicas con las necesidades del mercado y las prácticas organizacionales (Rothwell, 1991).

Evidentemente, la incertidumbre está presente tanto en la gestación de una innovación, como en su aceptación por parte del mercado; por consiguiente, la innovación se concibe como un proceso social donde las interacciones entre los agentes socioeconómicos constituyen un factor clave del éxito empresarial.

De esta incertidumbre no se escapan los ejercicios de medición de la innovación. En este sentido todos los países deben pregun- 
tarse cuál es la concepción de innovación que más se ajusta a sus características idiosincrásicas y, en consecuencia, cómo orientar los correspondientes ejercicios de medición. Surge por lo tanto un problema para los investigadores, ¿cómo orientar adecuadamente la medición de tal forma que se consiga información útil para las sociedades que se sirven de ella sin detrimento de la comparabilidad internacional?

\section{LA IMPORTANCIA DE LAS INTERACCIONES}

Las interacciones entre los agentes pueden ser considerados desde una doble funcionalidad: son esenciales para gestionar el complejo proceso de la innovación, y a la vez, la gestión de la innovación busca desarrollar las esenciales interacciones entre los agentes de una red o sistema productivo. De hecho, actualmente, uno de los vínculos más productivos son los establecidos entre universidad (o institutos de I+D) y empresa.

Dado que las actividades innovativas en las empresas se han profesionalizado y que la investigación universitaria se ha especializado, algunas universidades vienen incrementando el entrenamiento de sus investigadores sobre sectores de interés para las empresas, al mismo tiempo que las empresas se han dado cuenta de que los grandes programas investigativos de las universidades pueden traer beneficios para su innovación (Pavitt, 2003).

Pero, la relación industria-universidad suele ser difícil de gestionar. Los desacuerdos son de lado y lado, a menudo los gestores en las empresas argumentan una incompatibilidad entre los largos tiempos de trabajo universitario versus las urgencias de los negocios, y por su parte, las universidades se sienten consideradas sólo como una fuente de recursos a bajo costo explotadas las empresas.

$\mathrm{Al}$ respecto, estudios recientes (Pavitt, 2003) han mostrado la importancia de tres prácticas, complementarias entre sí, para fortalecer los vínculos entre la universidad y la innovación empresarial: 
- El fomento de los contactos personales, en muchas ocasiones, establecidos informalmente.

- Sensibilizar a las empresas sobre la utilidad de la investigación universitaria.

- Dar a conocer los beneficios prácticos de una buena parte de la investigación universitaria, ya que surge de la combinación entre nuevo conocimiento teórico, nuevas técnicas y observaciones, y nuevas habilidades, acciones que a las empresas les dificulta realizar por sí solas.

Otro aspecto que complementa la tríada básica para gestionar la innovación, con respecto a las interacciones sociales, es la relación con los programas de gobierno. Hoy existe una amplia gama de herramientas gubernamentales disponibles para la asistencia de los gestores en la formulación, financiación y desarrollo de proyectos.

Se conocen diversos tipos de experiencias al respecto, que incluso generan variadas posturas, al punto que se cuestiona el costo de oportunidad de estas iniciativas o programas gubernamentales, especialmente, cuando el soporte gubernamental trata de acelerar en forma crítica el aprendizaje tecnológico en un momento en el que el mercado no está listo para asumir ciertos riesgos.

Con el fin de hacer frente a esa complejidad se ha desarrollado una herramienta para el estudio y gestión de la innovación, los Sistemas de Innovación (Lundvall ed., 1992; Nelson y Rosenberg, 1993; Freeman, 1995; Edquist ed., 1997). Éstos son un marco de análisis mediante el cual se pretende conocer la estructura económica y social de un determinado territorio, fundamentándose, en gran medida, en la teoría del aprendizaje interactivo centrada en las relaciones existentes entre los diversos agentes que constituyen un Sistema de Innovación en particular. 


\section{LOS SISTERPMS DE INNOVMCIÓN}

La gestión de los procesos de aprendizaje y la creación de condiciones que favorecen la innovación se han convertido en un elemento central en el diseño de políticas, para lo cual se analizan tanto las experiencias propias como las de de otros países o regiones.

Ahora, dado que un sistema productivo es abierto, dinámico y social, como consecuencia de las interacciones no sólo entre los agentes socioeconómicos que lo constituyen, sino también, por las interacciones con el entorno, el enfoque de Sistema de Innovación pretende analizar la existencia e interacción de los agentes presentes en un determinado territorio (nación o estado, región, etc.) tales como instituciones gubernamentales, clusters, universidades, industrias, etc., además de conocer las competencias de éstos, con el fin de aportar a las autoridades (nacionales, regionales o locales) elementos de juicio que faciliten la gestión de la innovación (Lundvall ed., 1992).

Por esta razón, es fácil encontrar una amplia gama de proyectos de investigación en los que se estudian las relaciones que se establecen entre los agentes de los Sistemas de Innovación (European Planning Studies, Vol. 8, Not. 4, 2000). Además, se han creado diferentes modelos de simulación para tratar de medir las características de dichos Sistemas en diferentes entornos (Simulating Self-Organizing Innovation Networks -SEIN).

De hecho, es posible diferenciar tres esenciales líneas de actuación en las que se han desarrollado los Sistemas de Innovación (Zabala, 2004):

- Estudios basados en Políticas de Innovación, comparando las características de diferentes Sistemas de Innovación por medio de análisis de Benchmarking.

- Estudios que pretenden formalizar el concepto de los Sistemas Nacionales de Innovación a través de modelos descriptivos o analíticos. 
- Estudios sobre los Sistemas Nacionales y/o Regionales de Innovación de determinados países y/o regiones.

Sin embargo, aún existe un amplio campo por abordar en estos Sistemas, pudiéndose al menos citar tres posibles áreas objeto de estudio:

- En primer lugar, se requiere de una combinación más explícita entre el concepto de Sistemas Nacionales de Innovación y el crecimiento económico.

- En segundo lugar, la relación existente entre el Sistema de Innovación de un determinado país y el resto de los subsistemas (mercado de trabajo, sistemas financieros, etc.) está lejos de ser abordada de modo exhaustivo. Esta limitación es aún más relevante, al tratarse de sistemas abiertos, en donde su fortaleza depende entre otros aspectos, de su relación con varios sub-segmentos de la economía.

- Por ultimo, es preciso mencionar el limitado conocimiento del que se dispone de las propiedades dinámicas en los Sistemas de Innovación, especialmente en lo concerniente a su estabilidad y evolución estructural.

Es claro, entonces, que para abordar debidamente estos objetos de estudio, la innovación debe concebirse en el sentido amplio y complejo, propuesto en este artículo, como un proceso social e interactivo.

\section{LAS REDES DE INNOVACIÓN (INNOVATION NETWORKS)}

Tal como se ha insinuado a lo largo del artículo, las empresas pocas veces innovan de modo aislado y los agentes interactúan para fomentar, desarrollar e intercambiar diversos tipos de conocimiento, información, experiencias y otro tipo de recursos. En muchos casos, esta actividad innovadora se desarrolla basada en las competencias presentes en un territorio (Olazaran y Gómez Uranga eds., 2000). 
Sin embargo, debido al cambio que se está produciendo en el entorno socio-económico global, las interacciones entre diferentes regiones y países se están convirtiendo en algo cotidiano, por lo tanto, la introducción de nuevos agentes y la generación de nuevos tipos de interacciones son una de las características más marcadas en la dinámica de los sistemas, asunto que ha llevado a la necesidad de hablar de redes de interacción al interior y entre los sistemas, algo que se está denotando como Innovation Networks o Redes de Innovación. De hecho son un fenómeno relativamente reciente que emergió a comienzos de los años 90 y son consideradas como una herramienta útil para explicar algunos fenómenos tales como las dinámicas de las organizaciones empresariales y las de las industrias de un sistema productivo local (Vázquez, 1999).

Por ejemplo, de las redes de relaciones entre gobierno-empresa-universidad (lo que se ha denominado la Triple Hélice) puede surgir un nuevo régimen global, de tal forma, que la red resurge como una nueva unidad de evolución. Esto significa, que cuando una estructura en forma de red haya adquirido cierta estabilidad, podrá co-evolucionar con el sistema en su conjunto y sus dinámicas. Parece claro, entonces, que la generación de redes de interacción entre los agentes de un Sistema de Innovación es uno de los principales argumentos por los que éste evoluciona y se desarrolla. En este contexto, las redes representan un mecanismo para la difusión de innovaciones a través de colaboraciones y de relaciones interactivas (Zabala, 2004).

Por lo tanto, debido al papel que desempeñan las redes en un Sistema de Innovación eficiente, los lazos de unión entre sus agentes deben ser muy estrechos, ya que los procesos de generación y difusión de nueva tecnología descansan en cierto modo sobre la reducción de los costes de transacción a través de las redes. Así mismo, una Red de Innovación bien establecida puede contener una gran inercia y una gran dependencia de su trayectoria histórica y tecnológica (Carlsson y Jacobson, 1997; Archibugi, Howells y Michie eds., 1999). 
En este orden de ideas, las Redes de Innovación son un concepto poliédrico, dependiente de la perspectiva que el investigador adopte, por consiguiente, es creciente la necesidad de elaborar ciertas métricas que permitan efectuar mediciones en aquellos procesos relacionados con el establecimiento de redes y la evaluación de las Políticas de Innovación y, de esta forma, puedan ser comparados los resultados cuantitativos de las simulaciones con las medidas reales obtenidas. Esto no sólo mejoraría el comportamiento del modelo de simulación, sino que además se podría comprender mejor la dinámica del sistema analizado (Saviotti ed., 2003).

En efecto, la literatura filosófica, económica, sociológica, política, entre otras, ha comenzado a demostrar que los recientes desarrollos en la generación de nuevo conocimiento pueden ser conceptualizados en términos de Redes de Innovación o Innovation Networks. Pero, a pesar de esto, aún existe un largo camino por recorrer debido a la variedad de perspectivas que éstas permiten adoptar, asunto que ha inducido a la comunidad científica para desarrollar modelos de simulación que permiten determinar los posibles eventos que pudieran ocurrir en las redes y en los Sistemas de Innovación a los que pertenecen, para que los investigadores puedan considerar diferentes escenarios en el desarrollo de un Sistema.

\section{CONCHUSONEN}

Puede concluirse, entonces, que la innovación permanece impredecible en sus consecuencias técnicas, comerciales e incluso, sociales. Definitivamente para los gestores corporativos, su heterogeneidad y naturaleza contingente significa que no hay simplemente buenas prácticas en los modelos de innovación de las empresas, por lo tanto, en términos generales, se acepta que la producción de conocimiento, su distribución, así como el desarrollo social y económico son procesos complejos y directamente relacionados con las interacciones y dinámicas propias de las redes o sistemas productivos y sociales en que se hayan inmersas. 
En consecuencia, se sugiere asumir la innovación como un proceso social e interactivo, y que, mediante los ejercicios de medición, se identifiquen elementos de juicio orientadores del diseño e implementación de políticas públicas capaces de integrar los agentes socioeconómicos en torno a nuevos caminos de construcción de conocimiento.

En definitiva, la innovación debe ser considerada desde un enfoque más social, esto es, que no la defina simplemente como productos o procesos nuevos o mejorados, ni tampoco que la limite al entorno empresarial e institucional.

Ahora, para gestionar mejor la innovación, deben cualificarse los ejercicios de medición. Para lograr tal fin, es fundamental definir al menos dos determinantes: primero, cuál es el elemento aglutinante que determinará el espacio cultural por estudiar, por ejemplo: una región geográfica, una dinámica económica, un sector estratégico para el desarrollo, un sector en conflicto, etc., y segundo, deben expresarse conceptualmente cuáles son los elementos característicos de dicho espacio. Sin estos elementos de juicio, no será posible una adecuada definición de innovación y por consiguiente, su medición no será información útil para la formulación de políticas públicas.

En tal caso, la medición de la innovación debe ser una actividad científica, que permita ese acercamiento a los conceptos de ciencia, tecnología y su relación con la sociedad, así como esa búsqueda de métodos de gestión que orienten el cambio técnico hacia la innovación para el desarrollo social. Esta medición, basada en las relaciones o interacciones, determina que el significado de innovación no existe en sí mismo, sino que depende de su contexto de producción, en donde se contrasta y valida.

Consecuentemente, puede asegurarse que la forma como se mide la innovación actualmente en Latinoamérica padece de ciertas insuficiencias que "no dejan ver" detalles que resultan esenciales para alimentar los procesos de construcción de conocimiento, así como para formular y aplicar políticas públicas que logren sintonizar las capacidades productivas con las oportunidades y demandas del mercado. 


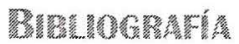

ARCHIBUGI, D., HOWELls, J., MICHIE, J. (eds.), Innovation Policy in a Global Economy. Cambridge: Cambridge University Press, 1999.

CARLSSON, B., JACOBSON, S. Diversity Creation and technological Systems: A Technology Policy Perspective. p. 266-294. En: EDQUIST, C. (ed.). Systems of Innovation: Technologies, Institutions and Organizations. London: Pinter, 1997.

EDQUIST, C. (ed.) Systems of Innovation: Technologies, Institutions and Organizations. London: Pinter, 1997.

EUROPEAN Planning Studies. Vol. 8, No. 4 (2000).

FREEMAN, C. The National System of Innovation in historical perspective. En: Cambridge Journal of Economics. No. 19 (1995); p.5-24.

LUNDVALL, B. A. (ed.) National Systems of Innovation. Towards a Theory of Innovation and Interactive Learning. London: Pinter, 1992.

NELSON, R.R., ROSENBERG, N. Technical innovation and national systems. p. 3-21. En: Nelson, R.R. (ed.). National Innovation Systems: A comparative Analysis. New York: Oxford University Press, 1993.

OLAZARAN, M. y GÓMEZ, Uranga M. (eds.). Sistemas Regionales de Innovación. Servicio Editorial de la Universidad del País Vasco, 2000.

PAVITT, Keith. The Process of Innovation, 2003. England: Science and Technology Policy Research SPRU, The Freeman Centre, University of Sussex. p. 47.

ROTHWELL, R. Successful Industrial Innovation: Critical Factors for the 1990s. Extended Version of a Paper Presented at the Science Policy Research Unit's 25 $5^{\text {Th }}$ Anniversary Conference: Perspectives on the Future of Science and Technology Policy. England: SPRU, University of Sussex, 1991.

SAVIOTTI, P.P. (ed.) Applied Evolutionary Economics: New Empirical Methods and Simulation Techniques. Cheltenham: Edward Elgar, 2003.

VÁZQUEZ BARQUERO, A. Desarrollo, redes e innovación: lecciones sobre desarrollo endógeno. Madrid: Ediciones Pirámide, 1999.

ZABALA, Jon Mikel, 2004. Análisis y medición de las interacciones en los Sistemas Regionales de Innovación. Su relación con la trayectoria histórica y tecnológica de las regiones. Memoria del Proyecto de Investigación para la obtención de la Suficiencia Investigadora del Programa de Doctorado de Proyectos de Ingeniería e Innovación. España: Universidad Politécnica de Valencia. 